\title{
Morphological traits and density of foundation species modulate a facilitation cascade in Australian mangroves
}

\author{
Melanie J. Bishop, ${ }^{1,3}$ Jill Fraser, ${ }^{1}$ and Paul E. Gribben ${ }^{2}$ \\ ${ }^{1}$ Department of Biological Sciences, Macquarie University, Sydney, New South Wales 2109 Australia \\ ${ }^{2}$ Plant Functional Biology and Climate Change Cluster and, School of the Environment, University of Technology, \\ Sydney, New South Wales 2007 Australia
}

\begin{abstract}
Facilitation cascades are critical to the maintenance of biodiversity in a variety of habitats. Through a series of two experiments, we examined how the morphological traits and density of interacting foundation species influence the establishment and persistence of a facilitation cascade in temperate Australian mangrove forests. In this system, mangrove pneumatophores trap the free-living alga, Hormosira banksii, which, in turn, supports dense and diverse assemblages of epifaunal mollusks. The first experiment, which manipulated pneumatophore height and density, revealed that these two traits each had additive negative effects on the establishment, but additive positive effects on the persistence of the cascade. High densities of tall pneumatophores initially served as a physical barrier to algal colonization of pneumatophore plots, but over the longer term enhanced the retention of algae. The increased algal biomass, in turn, facilitating epifaunal colonization. The second experiment demonstrated that the retention of algae by pneumatophores was influenced more by algal thallus length than vesicle diameter, and this effect occurred independent of pneumatophore height. Our study has extended facilitation theory by showing that the morphological traits and density of basal and intermediary facilitators influence both the establishment and persistence of facilitation cascades. Hence, attempts to use foundation species as a tool for restoration will require an understanding not only of the interactions among these, but also of the key traits that modify interrelationships.
\end{abstract}

Key words: density-dependence; ecosystem engineers; facilitation; foundation species; hierarchical facilitation; Hormosira banksii; mangrove; morphological traits; morphology; pneumatophores; positive interaction.

\section{INTRODUCTION}

The provision of biogenic habitat by foundation species (sensu Dayton 1972) is a key determinant of community assembly in many ecosystems (Jones et al. 1994, Bruno and Bertness 2001, Grutter and Irving 2007). Recently, there has been growing interest in how foundation species that co-occur in nested or adjacent assemblages cumulatively determine biodiversity (Thomsen et al. 2010, Angelini et al. 2011). One way is through facilitation cascades, whereby a basal foundation species enables the colonization of a second, which, in turn, facilitates the focal community (Bruno and Bertness 2001). Facilitation cascades have been identified across a range of ecosystems (e.g., tree-epiphytemobile epifauna, Ellwood et al. 2002; saltmarsh-musselepibiota, Altieri et al. 2007; mangrove-algae-epibiota, Bishop et al. 2012).

Studies on facilitation cascades have ascertained relationships between basal and intermediary facilitators, and the focal community through presence-

Manuscript received 24 October 2012; revised 20 March 2013; accepted 28 March 2013. Corresponding Editor: J. F. Bruno.

${ }^{3}$ E-mail: melanie.bishop@mq.edu.au absence (Altieri et al. 2007, Gribben et al. 2009) or density manipulations (Bishop et al. 2012). Consequently, we know little about the sensitivity of facilitation cascades to variation in morphological traits, such as size or shape, of the facilitators involved, or how these morphological traits of facilitators interact with their density, to influence the cascade. Foundation species can display considerable spatial and temporal variability in morphology and density (Gleason et al. 1979, Leonard and Luther 1995). Where these traits influence key functions of the foundation species, such as provision of substrate for attachment or amelioration of environmental stress, their facilitative role may be modified (e.g., Bruno and Kennedy 2000, van Hulzen et al. 2007, Irving and Bertness 2009, Harley and O'Riley 2011).

Traits of basal and intermediary facilitators might influence facilitation cascades in three ways. First, the morphology and density of the basal facilitator might determine whether the environment is suitable for the intermediary to colonize, and determine the density that the intermediary attains. This is critical because without the presence of the intermediary facilitator, perhaps at a threshold density (Bishop et al. 2012), a cascade cannot occur. Second, the morphology and density of basal facilitators may influence the morphological traits of 
successfully colonizing intermediaries. The morphological traits of the intermediaries may then, in turn, influence development of the focal community. Third, independent of the traits of the basal foundation species, variation in the morphology, and density of the intermediary may directly influence the focal community.

Within temperate mangroves of southeastern Australia, the pneumatophores of Avicennia marina support a facilitation cascade with the fucoid alga Hormosira banksii as intermediary (Bishop et al. 2012). The structure provided the pneumatophores traps free-living and reproductively capable $H$. banksii that washes into the mangrove forest following detachment from rocky shores (Plate 1, Appendix A; McKenzie and Bellgrove 2008). The alga, which is characterized by a distinctive thallus of branched chains of vesicles, in turn, supports dense and diverse communities of epifaunal mollusks (Plate 1, Appendix A; Bishop et al. 2009, 2012). Experiments independently manipulating the density of pneumatophores and the biomass of algae in a crossed design have shown that this indirect effect of pneumatophores on epifauna, arising via the cascade, overwhelms their direct effects (Bishop et al. 2012).

Pneumatophores can range in height from a few to tens of centimeters, and in density from a few to thousands per square meter (Bishop et al. 2007, Dahdouh-Guebas et al. 2007, Amaral et al. 2011). H. banksii vesicle diameter and length vary by as much as $20 \mathrm{~mm}$, and thallus length by tens of centimeters (Womersley 1987, Ralph et al. 1998, Macinnis-Ng et al. 2005). The morphology of the alga directly influences epifaunal colonization (Bishop et al. 2009), but it is unclear whether it also influences algal retention by pneumatophores.

Here, we describe two manipulative field experiments that address the sensitivity of this facilitation cascade to variation in the morphology and density of basal and intermediary facilitators. The first assesses how pneumatophore height and density interact to determine colonization and retention of the intermediary facilitator, algae, and the focal community, epifaunal mollusks. We hypothesized that although, initially, dense and tall pneumatophores will act as a physical barrier to algal colonization, over longer time periods, these traits will facilitate maintenance of greater algal biomass because once penetrating dense and tall pneumatophores, $H$. banksii is more effectively retained. Hence, we expected overall that pneumatophore height and density will positively influence the focal community. The second experiment assessed how pneumatophore height and algal morphology interact to influence the cascade. We hypothesized that, as vesicle diameter of the algae increases, a greater height of pneumatophores will be required to retain the algae because the buoyancy of the algae will increase. We hypothesized that as thallus length increases, however, a smaller pneumatophore height will be required for algal retention as the algae will be more easily entangled.

\section{Materials and Methods}

\section{Study site}

Experiments were conducted at Quibray Bay $\left(34^{\circ} 01^{\prime} 29^{\prime} \mathrm{S}, 151^{\circ} 10^{\prime} 45^{\prime} \mathrm{E}\right)$, within the Towra Point Aquatic Reserve, Botany Bay, Sydney, Australia. The first, which manipulated pneumatophore density and height, was done in the austral spring of 2011 (late August-October). The second, manipulating algal morphology and pneumatophore height was in the austral fall and winter of 2011 (April-August). Annual variation in water temperature at this site is typically $\sim 6^{\circ} \mathrm{C}$, and in contrast to many North American systems, invertebrates at this site do not display seasonal variation in abundance or richness (Bishop et al. 2007, 2009). The site was dominated by dense stands of the grey mangrove, Avicennia marina, at upper to mid intertidal elevations. Within the mangrove forest, freeliving Hormosira banksii was found trapped among pneumatophores, forming patches meters long and of up to $90-100 \%$ cover.

Manipulations of algal and pneumatophore traits were done on the unvegetated mudflat, 2-3 m seaward of the lower limit of pneumatophores of A. marina. This allowed us to nondestructively manipulate pneumatophore traits using structural mimics without being limited by the few gaps in pneumatophores cover in the mangrove forest. The pneumatophore mimics were $250 \mathrm{~mm}$ long and $6 \mathrm{~mm}$ wide bamboo chopsticks, inserted into the mud to achieve the desired height and density of structural elements (Appendix A). We have previously used these to investigate other aspects of the mangrove facilitation cascade and have demonstrated that these support similar biodiversity to real pneumatophores, higher on the shore (Bishop et al. 2012).

\section{Interacting effects of pneumatophores height and density on the facilitation cascade}

The design of the first experiment, manipulating the density and height of pneumatophores, included three levels of each factor, spanning the full range of morphological variation at our field site (see Bishop et al. 2012). For pneumatophore density, the levels were 50 (low, L), 100 (medium, M), and 150 (high, H) mimics/ $0.25 \mathrm{~m}^{2}$ plot. For pneumatophore height, they were 3 (short, S), 7 (intermediate, I), or 10 (tall, T) $\mathrm{cm}$. Additionally, we established a control treatment with no pneumatophores. Each of the 10 treatments had six replicate $0.5 \times 0.5 \mathrm{~m}$ plots.

The 60 plots, each separated by $1-2 \mathrm{~m}$, were established at a tidal height of mean low water $+0.6 \mathrm{~m}$. Each plot was randomly assigned to a treatment and chopsticks were inserted vertically into sediments to produce the assigned height and density of pneumatophore mimics. At the start of the experiment, each plot was surrounded by $8 \mathrm{~kg}$ wet mass of $H$. banksii 
transplanted from the mangrove forest to provide a similar frond density to observed accumulations of algae on sandy beaches following peak recruitment events (McKenzie and Bellgrove 2008). The alga was not placed in direct contact with the chopsticks and formed a complete circle around each plot. This ensured that, in the absence of treatment effects, there was equal opportunity for algae to be trapped by each plot. An additional $40 \mathrm{~kg}$ (wet mass) of $H$. banksii was introduced to the seaward side of the study site every two weeks to maintain supply.

On two days, 1, 2, 4, 6, and 8 weeks following the commencement of the experiment, we measured the wet mass of algae accumulated in each $0.25-\mathrm{m}^{2}$ plot. Algae were towel-dried, weighed using a $10-\mathrm{kg}$ spring-loaded balance, and then returned to their plot. At each sampling time, we also assessed whether the number and richness of the colonizing epifaunal mollusks were correlated to the biomass of algae that had accumulated. Mollusks were quantified within a $0.2 \times 0.2 \mathrm{~m}$ quadrat placed in the center of each plot, at least $0.1 \mathrm{~m}$ from the plot edge. We enumerated mollusks by species, summing across the three substrates to which they were attached: the algal canopy, the pneumatophores mimics, or the sediment. We first searched any $H$. banksii within the quadrat for mollusks. We then carefully moved the algae aside, and identified and counted mollusks on the pneumatophores mimics and sediment surface below. Algae and associated mollusks were returned to each plot following enumeration. This method of sampling does not modify established communities of mollusks (Bishop et al. 2009).

\section{Interacting effects of $\mathrm{H}$. banksii vesicle size and pneumatophore height on algal retention}

The second experiment, testing how the retention of $H$. banksii is influenced by the interacting effects of algal morphology and pneumatophore height, utilized five algal morphologies (Fig.1), and three pneumatophore heights (short [S], $3 \mathrm{~cm}$; intermediate [I], $7 \mathrm{~cm}$; tall [T], 10 $\mathrm{cm}$; as per experiment 1), in a crossed design. The five algal morphs were from different mangrove forests along the New South Wales coast and each differed in their vesicle size and/or thallus length (Fig. 1). As thallus length generally increases with vesicle diameter (Fig. 1), we did not attempt to independently manipulate these two traits. The experimental design also included three control treatments (one for each pneumatophore height) that were free of algae. Control plots assessed the rate at which new algae, which was not experimentally manipulated, washed into plots of differing pneumatophore height. This allowed us to determine whether assessments of $H$. banksii retention were confounded by the arrival of new material.

A total of 108 plots, $0.25 \mathrm{~m}^{2}$ in area, were marked with a single polyvinyl chloride (PVC) stake and assigned to one of the 18 treatments, to give $n=6$ replicates for each. Each plot received 100 pneumato-

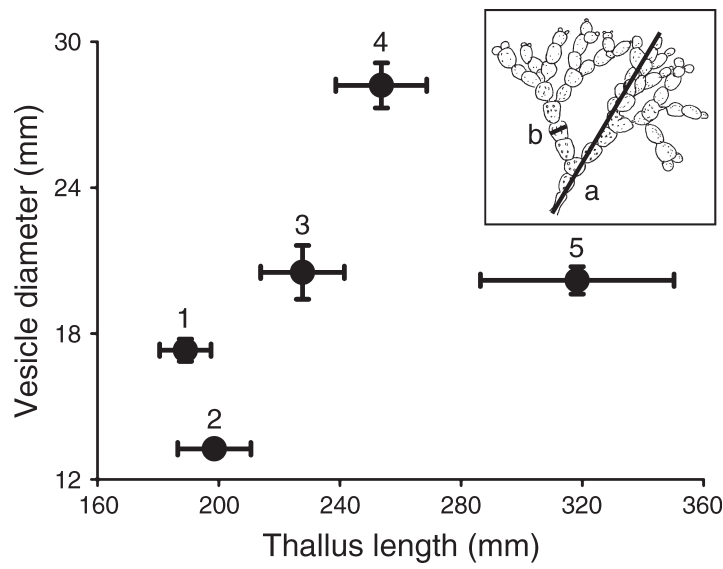

FIG. 1. Frond (thallus) length (see inset, line a; mean \pm SE, $n=20$ ) and vesicle diameter (see inset, line $\mathrm{b}$; mean $\pm \mathrm{SE}, n=12$ means, each calculated from five vesicles per frond) of each of the five algal morphs of Hormosira banksii (numbered 1-5) used in the experiment. One-way ANOVA revealed that morph 5 had significantly longer fronds than any of the other four morphs, which, in turn, did not significantly differ in frond length from one another (ANOVA, $F_{4,95}=10.1, P<0.001$; Student-Newman-Keuls [SNK], $5>[1=2=3=4])$. The vesicle diameters of morphs could be separated into four distinct groups, with morph 4 comprising the largest vesicles, and morph 2 the smallest (ANOVA, $F_{4,55}=58.0, P<0.001$; SNK, 4 $>[3=5]>1>2)$.

phore mimics, depressed to give the required height. The plots assigned to an algal treatment were supplied with 2 $\mathrm{kg}$ wet mass of defaunated $H$. banksii of the designated morphology. This pneumatophore density and algal biomass were based upon the average per $0.25-\mathrm{m}^{2}$ quadrat in the adjacent mangal. $H$. banksii was evenly spread across the $0.25-\mathrm{m}^{2}$ area of designated plots at low tide. $H$. banksii was not fenced in quadrats, thus allowing for its biomass to adjust naturally.

To assess retention of $H$. banksii, we measured the wet mass of $H$. banksii remaining in each experimental plot one, two, and four months after experimental addition. At each sampling time, we also quantified colonizing mollusks. Methods were as described in the previous subsection (Interacting effects of pneumatophores height and density on the facilitation cascade).

\section{Statistical analysis}

We analyzed data from each sampling time separately to avoid issues of nonindependence associated with taking repeated measurements from the same experimental plots (see Underwood 1997). All analyses were run on three key variables of interest: (1) algal biomass, (2) molluscan abundance, and (3) molluscan richness. Analyses of variance (ANOVAs) were run using GMAV5 (University of Sydney, Sydney, Australia). Correlations were run in Minitab (version 16; Minitab 2010).

Data from the first experiment were analyzed using asymmetrical ANOVAs that simultaneously tested for 

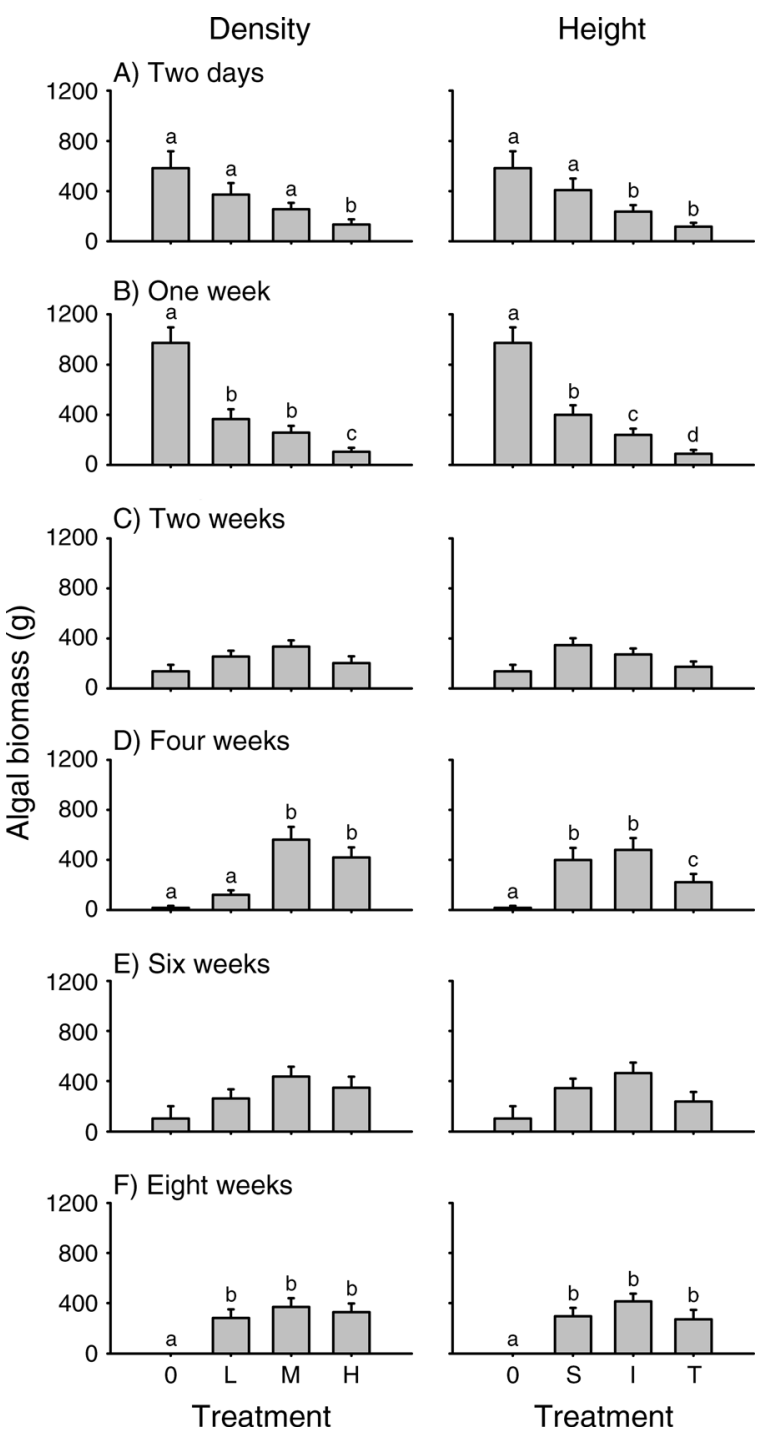

FIG. 2. Biomass of algae (mean $+\mathrm{SE})$ in plots of low $(\mathrm{L}, 50$ mimics $/ 0.25 \mathrm{~m}^{2}$ ), medium (M, $100 \operatorname{mimics} / 0.25 \mathrm{~m}^{2}$ ), or high density $\left(\mathrm{H}, 150\right.$ mimics $\left./ 0.25 \mathrm{~m}^{2}\right)$ of pneumatophore mimics, and of short $(\mathrm{S}, 3 \mathrm{~cm})$, intermediate $(\mathrm{I}, 7 \mathrm{~cm})$, or tall $(\mathrm{T}, 10 \mathrm{~cm})$ height ( $n=18$ plots per treatment). Control plots $(0, n=6)$ were free of mimics. Effects of density and height are considered independently because ANOVAs indicated no interaction between these factors. Lowercase letters above the bars denote levels of significant treatment effects (ANOVA, Appendix B: Table B1) that statistically differed at $\alpha=0.05$ (SNK tests).

(1) differences in the variables of interest between structureless control plots and those with pneumatophore mimics, and (2) effects of pneumatophore density and height. These analyses involved partitioning components of variation through a number of sub-analyses (see Winer 1971). First, we ran one-way analyses testing for differences among all 10 treatments (nine different pneumatophore treatments and the control). Second, we ran two-way analyses, including only the pneumatophore treatments, testing for effects of density and height. We then subtracted variation among pneumatophore treatments from variation among all treatments to test for effects of pneumatophores that occurred irrespective of height or density. Where Cochran's tests indicated heterogeneity of variances among raw data, $\ln (x+1)$-transformed data were analyzed. Where ANOVA indicated that factors were significant at $\alpha=$ 0.05 , the tests was followed by post hoc StudentNewman-Keuls (SNK) tests to assess sources of treatment differences. Pearson's correlations tested for relationships between algal biomass and epifaunal abundance or richness at each of the six sampling times.

Data from the second experiment, on the sensitivity of the facilitation cascade to algal morphology and pneumatophore height, were also analyzed using asymmetrical ANOVAs. These first compared control plots to those with algal morphs, before comparing algal morphs.

\section{RESULTS \\ Interacting effects of pneumatophore height and density on the facilitation cascade}

At each sampling time, there was no interactive effect of pneumatophore density and height on algal biomass (Appendix B: Table B1). Hence, the main effects of density and height were additive.

Effects of pneumatophore height and density varied through time (Fig. 2; Appendix B: Table B1). After two days and one week, control plots, without pneumatophore mimics, contained a greater biomass of $H$. banksii than any of the experimental treatments, although this pattern was not statistically significant by the two day sampling (Appendix B: Table B1). Among experimental treatments, algal biomass decreased with increasing pneumatophore height and density (Fig. 2A, B). By four weeks, however, the control plots contained significantly less algae than all but the low pneumatophore density treatment (Appendix B: Table B1). At four weeks, the medium and high densities of pneumatophores supported a greater biomass of algae than the low density, and the small and intermediate heights supported more algae than the tall height (Fig. 2D). In weeks 6 and 8 there were no statistically significant difference among levels of either the pneumatophore height or density treatment (Appendix B: Table B1), although by week 8 each of the plots with pneumatophore mimics contained more alga than control plots (Fig. 2F).

Seven different species of mollusk colonized experimental plots. These were: Salinator fragilis $(50 \%$ of all colonizing individuals), Bembicium auratum (31\%), Austrocochlea porcata (8\%), Batillaria australis (5\%), Pyrazus ebeninus (3\%), Polinices sodidus (1\%), and Nassarius burchardi (1\%). Initially, two days after the start of the experiment, the number of mollusks in plots was small and there was little difference in their abundance among experimental treatments or between treatments and controls (Fig. 3A; Appendix B: Table 
B2). As colonization of plots progressed, and abundances increased, differences emerged. By one week, a pattern of greater molluscan abundance at the intermediate pneumatophore height than in any of the other height treatments or the controls was evident, although there was no statistically significant effect of pneumatophore density (Fig. 3B; Appendix B: Table B2). By two weeks, experimental treatments with pneumatophore mimics contained more mollusks than control plots, irrespective of the height or density of mimics (Fig. 3C; Appendix B: Table B2;). This pattern of fewest mollusks in the control plots persisted throughout the experiment, but differences among experimental treatments emerged (Fig. 3; Appendix B: Table B2). Main effects of pneumatophore density and height on molluscan abundance were evident at four, six, and eight weeks (Fig. 3D-F; Appendix B: Table B2). Generally, plots with the medium or high density of mimics, or of intermediate or tall height, contained greater numbers of mollusks than plots with the low density or short height of mimics. In many instances, plots with the low density or short height of pneumatophore mimics were statistically indistinguishable from the control plots (Fig. 3DF).

Differences among treatments in the species richness of mollusks developed rapidly and culminated in a main effect of pneumatophore density, but not of pneumatophore height, on the number of taxa present (Fig. 4). By one week, plots with the intermediate height of pneumatophores contained more species than any of the other treatments, which, in turn, were statistically indistinguishable from one another (Appendix B: Table B3). As time progressed, the plots with pneumatophore mimics were colonized by more species of mollusk than control plots. A positive relationship between the density of mimics and the richness of mollusks developed (Fig. 4; Appendix B: Table B3), but the effect of height of pneumatophores was seldom important in determining species richness (Fig. 4; Appendix B: Table B3).

Shortly after the start of the experiment, the relationship between Hormosira biomass and each of molluscan abundance and richness was weak. As colonization progressed, however, relationships strengthened and were statistically significant from two weeks through to the end of the experiment (Fig. 5).

\section{Interacting effects of $\mathrm{H}$. banksii vesicle size and pneumatophore height on algal retention}

At each of the sampling times, one, two, and four months following establishment of experimental treatments, control plots, initially free of algae, contained negligible biomass of Hormosira (Fig. 6A; Appendix C: Table C1). Further, in not one instance did we observe the biomass of algae in a plot to increase between sampling times. Consequently, among the plots receiving one of the five algal morphs, measurements of algal
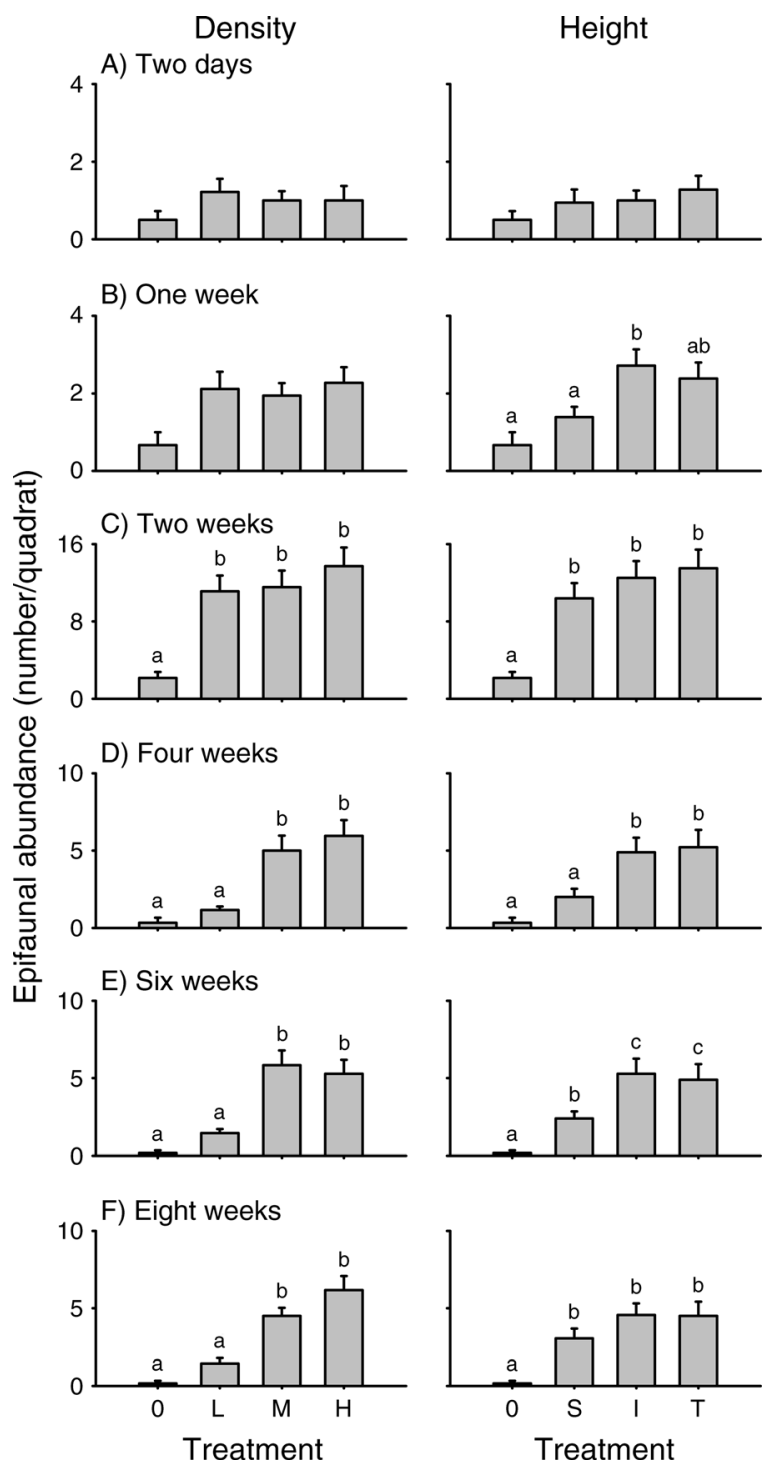

FIG. 3. Abundance (mean $+\mathrm{SE}$ ) of epifaunal mollusks per 0.04- $\mathrm{m}^{2}$ quadrat in plots of low $\left(\mathrm{L}, 50 \mathrm{mimics} / 0.25 \mathrm{~m}^{2}\right)$, medium (M, 100 mimics $/ 0.25 \mathrm{~m}^{2}$ ) or high $\left(\mathrm{H}, 150\right.$ mimics $\left./ 0.25 \mathrm{~m}^{2}\right)$ density of pneumatophore mimics, and of short $(\mathrm{S}, 3 \mathrm{~cm})$, intermediate $(\mathrm{I}, 7 \mathrm{~cm})$, or tall $(\mathrm{T}, 10 \mathrm{~cm})$ height $(n=18$ plots per treatment). Control plots $(0, n=6)$ were free of mimics. Effects of density and height are considered independently because ANOVAs indicated no interaction between these factors. Lowercase letters above the bars denote levels of significant treatment effects (ANOVA, Appendix B: Table B2) that statistically differed at $\alpha=0.05$ (SNK tests).

retention were not confounded by the arrival of new material.

Sampling one month after addition of algae to designated plots revealed that most of the 2-kg biomass had been retained (Fig. 6A). By four months following addition, however, on average, less than one-quarter of the material remained (Fig. 6A). At each of the sampling times, retention of algal biomass varied among morphologies (Appendix C: Table C1). Across all time 

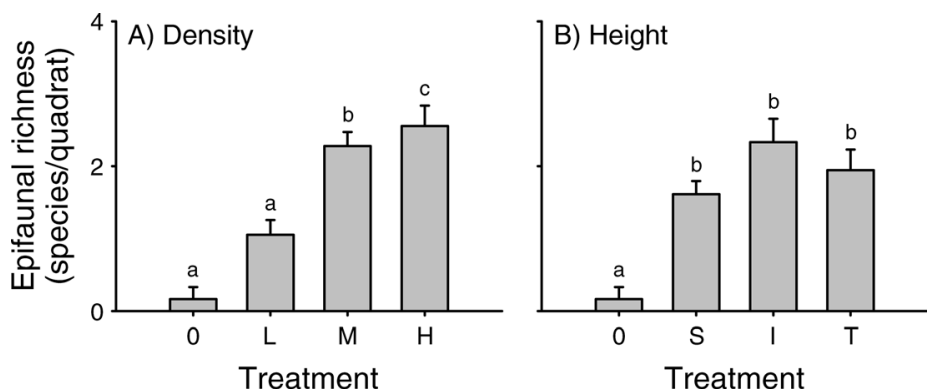

FIG. 4. Species richness (mean $+\mathrm{SE}$ ) of epifaunal mollusks per $0.04-\mathrm{m}^{2}$ quadrat at the end of the eight-week experiment in plots of low ( $\mathrm{L}, 50$ mimics $\left./ 0.25 \mathrm{~m}^{2}\right)$, medium $\left(\mathrm{M}, 100\right.$ mimics $\left./ 0.25 \mathrm{~m}^{2}\right)$, or high $\left(\mathrm{H}, 150\right.$ mimics $\left./ 0.25 \mathrm{~m}^{2}\right)$ density of pneumatophore mimics, and of short $(\mathrm{S}, 3 \mathrm{~cm})$, intermediate $(\mathrm{I}, 7 \mathrm{~cm})$, or tall $(\mathrm{T}, 10 \mathrm{~cm})$ height $(n=18$ plots per treatment). Control plots $(0, n=6)$ were free of mimics. Effects of density and height are considered independently because ANOVAs indicated no interaction between these factors. Lowercase letters above the bars denote levels of significant treatment effects (ANOVA, Appendix B: Table B3) that statistically differed at $\alpha=0.05$ (SNK tests).

scales, the greatest retention was in plots receiving morph 5, of longest thallus length (Fig. 6A; Appendix C: Table C1). Among the other morphs, which significantly differed in vesicle diameter but not frond length, differences in retention varied among sampling times (Fig. 6A). The retention of morphs 2 and 4 , which had the smallest and largest vesicle diameter, respectively, did not significantly differ at two of the three sampling times (Fig. 6A). Independent of algal morphology, pneumatophore height influenced retention of algal material. More algae were retained in plots with tall or intermediate pneumatophores mimics than with short mimics across the duration of the experiment (Fig. 6A).

Treatment effects on mollusks were temporally variable (Fig. 6B, C; Appendix C: Table C2). Initially, one and two months after algal addition to plots, fewer mollusks of fewer species were found in the control plots than in the plots receiving algal morphs (Fig. 6B, C). Among plots with algal morphs, molluscan abundance and richness did not follow patterns of algal retention. Instead, there were idiosyncratic patterns of difference in the abundance and richness of mollusks that varied among sampling times. Among some of the morphs, mollusks were more abundant in the plots with pneumatophores of intermediate or tall height. Nevertheless, this pattern was highly variable among algal treatments. Although the overall abundance and richness of mollusks increased with the amount of time available for colonization, treatment effects weakened (Fig. 6B, C; Appendix C: Table C2).

\section{DisCUSSION}

Previous studies have highlighted how traits of foundation species can modify their effects on associated organisms (Bruno and Kennedy 2000, Bruno 2002, van Hulzen et al. 2007, Irving and Bertness 2009, Harley and O'Riley 2011). Our study examined how the morphology and density of basal and intermediary facilitators interact to influence facilitation cascades. We have shown that in determining the density of intermediary foundation species, the morphology of basal foundation species can modify facilitation cascades. We have also provided evidence that the morphology of the intermediary foundation species can influence focal communities not only directly, but also indirectly by influencing the interaction between basal and intermediary facilitators.

\section{Cascading effects of basal species traits}

By manipulating pneumatophore density and height in an environment of algal supply, we found that the establishment of the facilitation cascade involving pneumatophores, the alga $H$. banksii and epifaunal mollusks was dependent on the density and height of pneumatophores. Further, we found that these traitmediated indirect effects were specific to stages in the
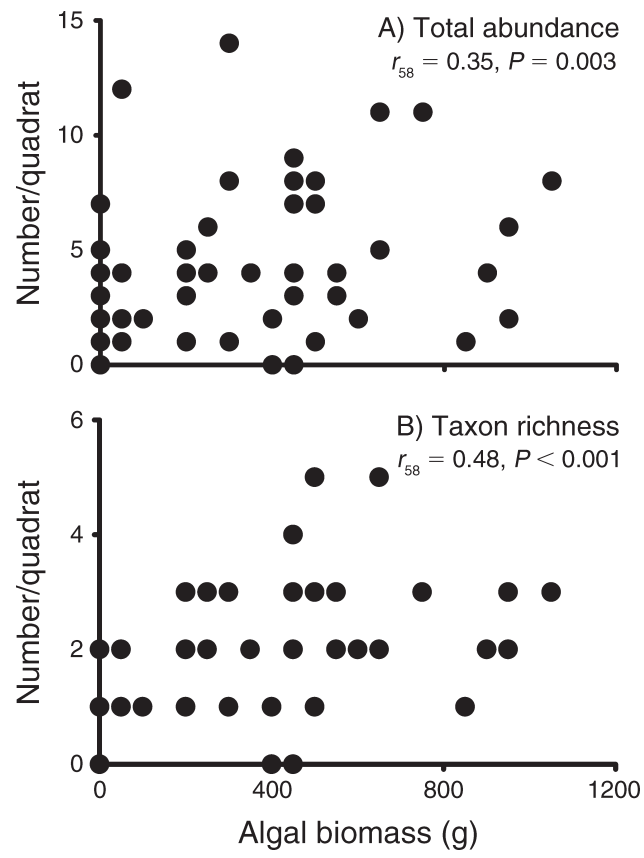

FIG. 5. Correlations between algal biomass and epifaunal mollusk (A) abundance and (B) richness at the end of the eightweek experiment ( $n=60$ plots). 

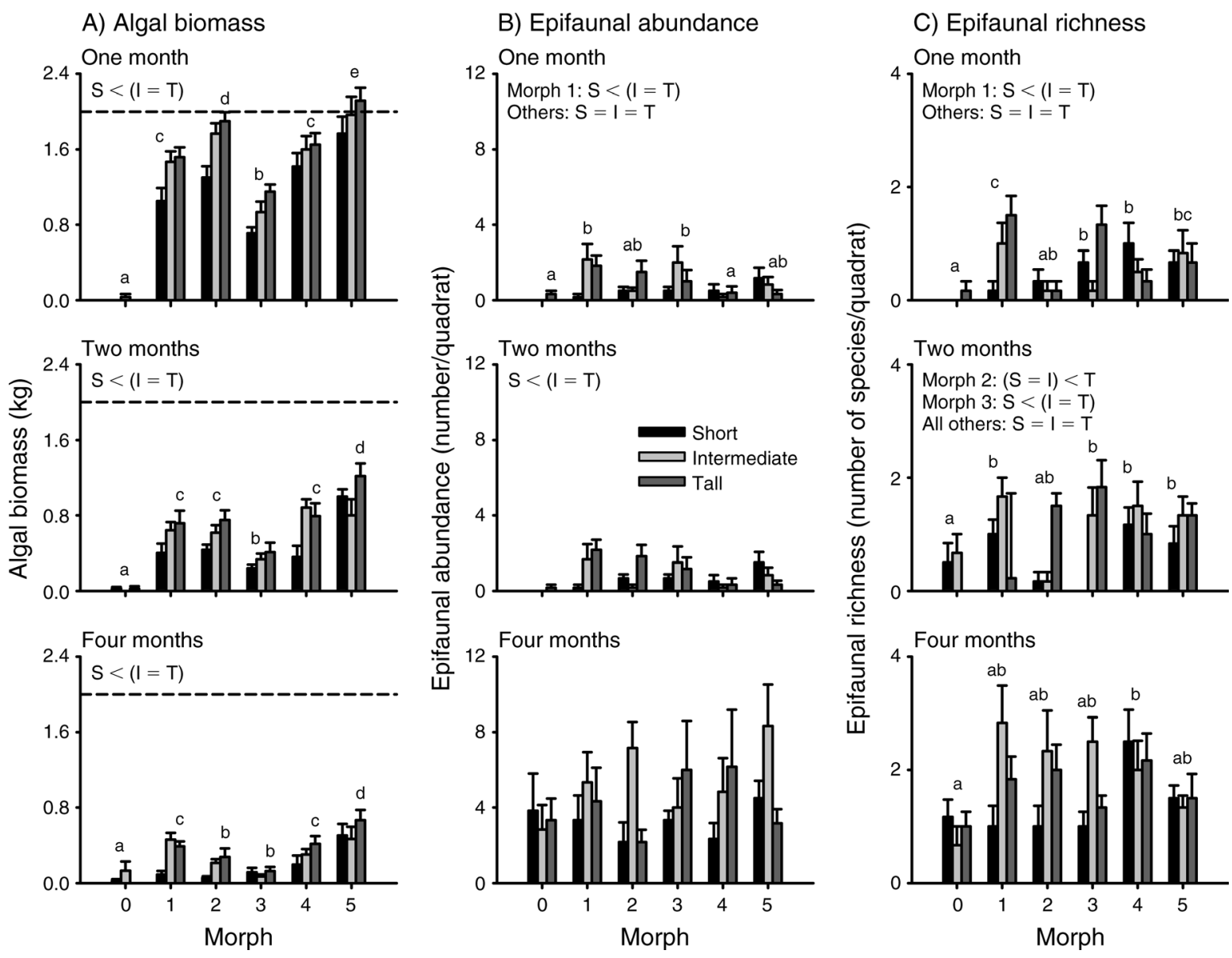

FIG. 6. (A) Algal biomass, (B) abundance of epifaunal mollusks, and (C) richness of epifaunal mollusks in experimental plots containing short $(\mathrm{S}, 3 \mathrm{~cm}$; black), intermediate (I, $7 \mathrm{~cm}$; light gray), or tall $(\mathrm{T}, 10 \mathrm{~cm}$; dark gray) pneumatophore mimics, at a density of $100 / 0.25-\mathrm{m}^{2}$ quadrat, that were initially free of algae (controls, 0 ) or that were supplied with $2 \mathrm{~kg}$ (dashed line) of one of five algal morphs (1-5 [see Fig. 1]; $n=6$ ). Histogram bars are means + SE. Plots were sampled on three occasions: one, two, and four months after the start of the experiment. Capital letters are shown on plots where there was an effect of pneumatophore height on the variable of interest and denote levels of the factor (S, I, T) that significantly differed (at $\alpha=0.05$ ) according to SNK tests (ANOVA; Appendix C). Where there was a main effect of algal morphology on the variable of interest, levels of the factors that statistically grouped together are indicated with lowercase letters (a-e) above the bars.

development of the facilitation cascade. Effects of pneumatophore height and density on algae and invertebrates were additive, not interactive. This was as predicted given the similar mechanisms by which morphology and density influenced both algal colonization and retention.

Initially, tall or dense pneumatophores acted as a physical barrier to algal transport, inhibiting algal colonization of pneumatophore patches by algae relative to plots that were bare or had few, small pneumatophores (Fig. 7). Hence, days to a week following the supply of algae to initially barren plots of pneumatophore mimics, $H$. banksii was negatively correlated with the density and height of pneumatophores. As time progressed, however, the relationship between pneumatophore traits and algal biomass shifted (Fig. 7). By one month, following establishment of barren plots, algal biomass was greater in plots with than without the alga, matching the pattern seen in mangrove forests with established H. banksii populations (Bishop et al. 2012). The change in the relationship was caused by the greater retention of algae by plots where there were dense (Bishop et al. 2012) or tall (second part of this study) pneumatophores. Although tall and dense pneumatophores were initially an impediment to $H$. banksii colonization as time progressed, it was the retention, not the colonization, of algae that was the limiting factor (Fig. 7).

Although more Hormosira was initially present in bare patches of sediment or those populated with less dense or short pneumatophore mimics, the abundance and richness of the focal community, epifaunal mollusks, did not follow this pattern. Instead, a similarly low abundance and richness of mollusks was initially found across all plots and no relationship between algae and mollusks was seen. As the time available for 


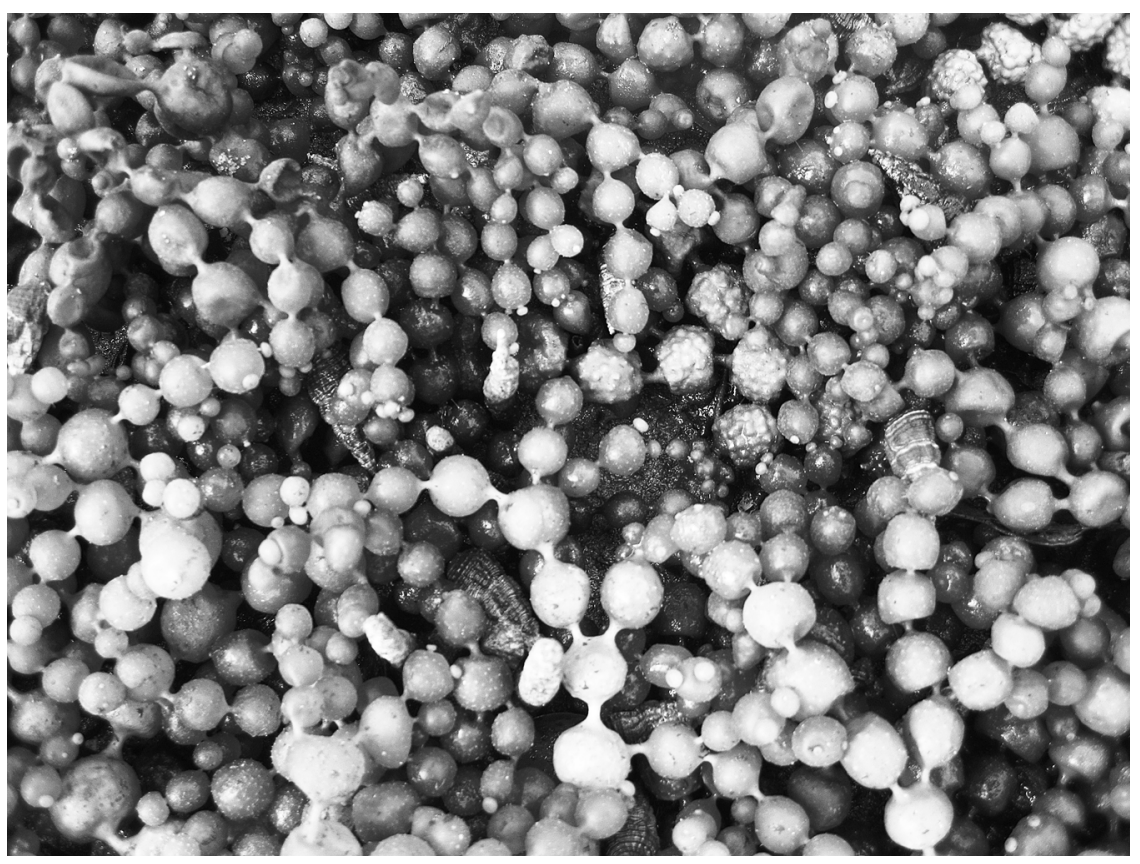

Plate 1. Following entanglement in mangrove pneumatophores, the complex structure of Hormosira banksii supports dense and diverse communities of invertebrates. Photo credit: J. Fraser.

recolonization of the initially defaunated plots by epifaunal mollusks increased, however, effects of pneumatophore density and height on mollusks emerged. Both algae and mollusks were more abundant in plots with dense or tall pneumatophores and the intermediary foundation species and focal community were, in turn, positively correlated to one another. Coupled with our previous demonstration of strong causation between algal abundance and the community structure of epifaunal mollusks over time scales of months (Bishop et al. 2012), this strong correlation at the latter sampling times suggested enactment of the facilitation cascade, with pneumatophores as basal foundation species, and the alga as the intermediary. Failure of the facilitation cascade to be enacted during the earlier stages of our experiment highlights the critical role that faunal colonization plays (see also Bishop et al. 2012).

\section{Indirect effects of intermediary facilitator traits}

Previous manipulations of $H$. banksii morphology have demonstrated that greater densities and diversities of mollusks are supported by algae with large than small vesicles (Bishop et al. 2009). This may arise as a result of the increased area of hard substratum for gastropod attachment and grazing of algal biofilms in this sedimentary environment where the availability of food resources can be limiting (Branch and Branch 1980, Byers 2000). Alternatively, the morphs may differ in their efficacy in protecting invertebrates from the abundant finfish and naticid predators in this environ-

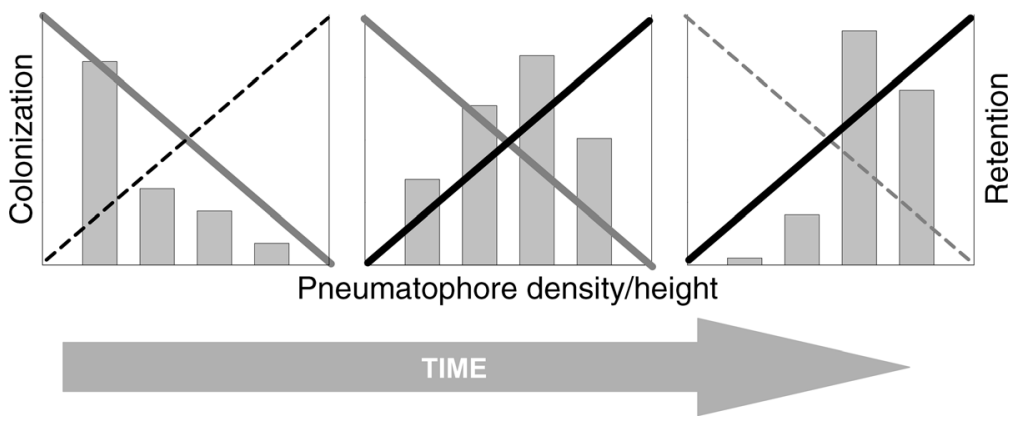

FIG. 7. Conceptual model showing how pneumatophore density and height have opposing effects on the colonization and retention of algae by experimental plots, and how the relative importance of these two factors changes over time to modify the relationship between pneumatophore density/height and algal biomass. Gray lines represent the relationship between pneumatophore density/height and algal colonization, and black lines show the relationship between pneumatophore height/ density and algal retention. Dashed lines are used to indicate weak effects, and solid lines show stronger effects. 
ment (Bayliss 1986, Anderson and Connell 1999), or in the size and shape of interstices they provide as habitat (see Hacker and Steneck 1990). This represents a direct effect of the intermediary facilitator on the focal community, of the type demonstrated by studies on individual foundation species (van Hulzen et al. 2007, Irving and Bertness 2009, Harley and O'Riley 2011). Here, we assessed whether there might also be an indirect effect of algal morphology arising from its effect on retention by pneumatophores. We found significant differences in the retention of the five algal morphs considered, with the morph of longest thallus consistently most effectively retained by pneumatophores, irrespective of pneumatophore height. Yet, among shorter morphs, thallus length was not a good predictor of retention and vesicle diameter bore no relationship to algal retention. Possibly, a longer thallus assists the retention of the algae by providing greater entanglement in the matrix of pneumatophores, but a threshold length is required. Other morphological traits, such as branching, were not considered here, but may help to explain variation.

Despite clear effects of algal morphology and pneumatophore height on algal retention, the relationship between algal biomass and the community structure of epifaunal mollusks in this second experiment was weak. This was counter to the positive relationship between algal biomass and molluscan communities in the first experiment, and in our previous research (Bishop et al. 2012). One and two months after the start of the experiment, plots with algal morphs generally had a greater abundance and richness of mollusks than control plots without algae, but among the plots with algae, differences in molluscan communities among treatments were idiosyncratic and unrelated to their algal retention. This suggests that either (1) traits of basal or intermediary facilitators, other than those manipulated, played a more important role in influencing epifauna; or (2) among plots with algae, molluscan colonization, not the availability of suitable habitat was the limiting factor. By three months, considerably greater molluscan recolonization of plots had occurred, but treatment effects on mollusks had weakened. This may be because algal biomass within even the best performing plots had dropped below 0.5 $\mathrm{kg}$, the critical threshold for the facilitation cascade to be enacted (Bishop et al. 2012).

\section{Implications}

In order for a facilitation cascade to be enacted, an intermediary foundation species must first colonize and persist in a habitat maintained by the basal foundation species, and the focal community must then colonize and persist in the habitat provided by the intermediary. Here we have shown that the morphology and density of either basal or intermediary foundation species that modify the ability of the associated communities to colonize or persist influence the establishment of facilitation cascades. We focused on how variation in the physical structure (i.e., density, size, shape) of basal and intermediaries might modify relationships. However, it is conceivable that variation in chemical traits (e.g., production of chemical cues for settlement, secondary metabolites that deter herbivory, and antimicrobial compounds) would also play a similar role. We have also shown how the same traits might affect multiple stages in the establishment of facilitation cascade in opposing ways. Increases in the density and height of the basal foundation species, pneumatophores, negatively influenced the colonization of the intermediary, $H$. banksii, yet positively influenced its persistence. The net effect of morphology and density on the facilitation cascade will hence be determined by the time scale of consideration. Our study adds to facilitation theory by showing that facilitation cascades must be defined not only by the basal and intermediary foundation species involved, but also by their morphological and density traits that allow for establishment and persistence of the cascade. Hence, attempts to use foundation species as a tool for restoration will require an understanding not only of the interactions among these (Halpern et al. 2007), but also how traits of basal and intermediary facilitators modify interrelationships.

\section{ACKNOWLEDGMENTS}

B. Coates and S. Towler assisted in the field. This research was supported by a Macquarie University Start-up Grant to M. Bishop. The comments of two anonymous reviewers improved the quality of the manuscript.

\section{Literature Cited}

Altieri, A. H., B. R. Silliman, and M. D. Bertness. 2007. Hierarchical organization via a facilitation cascade in intertidal cordgrass bed communities. American Naturalist 169:195-206.

Amaral, V., H. N. Cabral, and M. J. Bishop. 2011. Effect of runoff from acid sulfate soils on pneumatophores of the grey mangrove, Avicennia marina. Marine and Freshwater Research 62:974-979.

Anderson, M. J., and S. D. Connell. 1999. Predation by fish on intertidal oysters. Marine Ecology Progress Series 187:203211.

Angelini, C., A. H. Altieri, B. R. Silliman, and M. D. Bertness. 2011. Interactions among foundation species and their consequences for community organization, biodiversity and conservation. BioScience 61:782-789.

Bayliss, D. E. 1986. Selective feeding on bivalves by Polinices alderi (Forbes) (Gastropoda). Ophelia 25:33-47.

Bishop, M. J., J. E. Byers, B. J. Marcek, and P. E. Gribben. 2012. Density-dependent facilitation cascades determine epifaunal community structure in temperate Australian mangroves. Ecology 93:1388-1401.

Bishop, M. J., B. P. Kelaher, S. M. Sharp, and M. Verhoeven. 2007. Dispersal of the estuarine gastropod Pyrazus ebeninus is only weakly influenced by pneumatophore density. Journal of Experimental Marine Biology and Ecology 340:62-69.

Bishop, M. J., T. Morgan, M. A. Coleman, B. P. Kelaher, L. Hardstaff, and R. Evenden. 2009. Facilitation of molluscan assemblages in mangroves by the fucalean alga, Hormosira banksii. Marine Ecology Progress Series 392:111-122.

Branch, G. M., and M. L. Branch. 1980. Competition in Bembicium auratum (Gastropoda) and its effects on micro- 
algal standing stocks in mangrove muds. Oecologia 46:106114.

Bruno, J. F. 2002. Causes of landscape-scale rarity in cobble beach plant communities. Ecology 83:2304-2314.

Bruno, J. F., and M. D. Bertness. 2001. Habitat modification and facilitation in benthic marine communities. Pages 201218 in M. D. Bertness, M. E. Hay, and S. D. Gaines, editors. Marine community ecology. Sinauer, Sunderland, Massachusetts, USA.

Bruno, J. F., and C. W. Kennedy. 2000. Patch-size dependent habitat modification and facilitation on New England cobble beaches by Spartina alterniflora. Oecologia 122:98-108.

Byers, J. E. 2000. Competition between two estuarine snails: implications for invasions of exotic species. Ecology 81:12251239.

Dahdouh-Guebas, F., J. G. Kairo, R. De Bondt, and N. Koedam. 2007. Pneumatophore height and density in relation to micro-topography in the grey mangrove Avicennia marina. Belgian Journal of Botany 140:213-21.

Dayton, P. K. 1972. Toward an understanding of community resilience and the potential effects of enrichments to the benthos at McMurdo Sound, Antarctica. Pages 81-95 in B. C. Parker, editor. Proceedings of the colloquium on conservation problems in Antarctica. Allen Press, Lawrence, Kansas, USA.

Ellwood, M. D. F., A. T. Jones, and W. A. Foster. 2002. Canopy ferns in lowland dipterocarp forest supports a prolific abundance of ants, termites, and other invertebrates. Biotropica 34:575-83.

Gleason, M. L., D. A. Elmer, N. C. Pien, and J. S. Fisher. 1979. Effects of stem density upon sediment retention by salt marsh cord grass, Spartina alterniflora Loisel. Estuaries 2:271-273.

Gribben, P. E., J. E. Byers, M. Clements, L. A. McKenzie, P. D. Steinberg, and J. T. Wright. 2009. Behavioural interactions between ecosystem engineers control community species richness. Ecology Letters 12:1127-1136.

Grutter, A. S., and A. D. Irving. 2007. Positive interactions in marine communities. Pages 110-137 in S. D. Connell and B. M. Gillanders, editors. Marine ecology. Oxford University Press, South Melbourne, Australia.

Hacker, S. D., and R. S. Steneck. 1990. Habitat architecture and the abundance and body-size-dependent habitat selection of a phytal amphipod. Ecology 71:2269-2285.

Halpern, B. S., B. R. Silliman, J. D. Olden, J. P. Bruno, and M. D. Bertness. 2007. Incorporating positive interactions in aquatic restoration and conservation. Frontiers in Ecology and the Environment 5:153-160.
Harley, C. D., and J. L. O'Riley. 2011. Non-linear densitydependent effects of an intertidal ecosystem engineer. Oecologia 166:531-41.

Irving, A. D., and M. D. Bertness. 2009. Trait-dependent modification of facilitation on cobble beaches. Ecology 90: $3042-3050$

Jones, C. G., J. H. Lawton, and M. Shachak. 1994. Organisms as ecosystem engineers. Oikos 69:373-386.

Leonard, L. A., and M. E. Luther. 1995. Flow hydrodynamics in tidal marsh canopies. Limnology and Oceanography 40: $1474-1484$.

Macinnis-Ng, C. M. O., D. A. Morrison, and P. J. Ralph. 2005. Temporal and spatial variation in the morphology of the brown macroalga Hormosira banksii (Fucales, Phaeophyta). Botanica Marina 48:198-207.

McKenzie, P. F., and A. Bellgrove. 2008. Dispersal of Hormosira banksii (Phaeophyceae) via detached fragments: reproductive viability and longevity. Journal of Phycology 44:1108-1115.

Minitab. 2010. Minitab statistical software, version 16. Minitab, State College, Pennsylvania, USA.

Ralph, P., D. Morrison, and A. Addison. 1998. A quantitative study of morphological variation within Hormosira banksii (Turner) Decaisne (Phaeophyta: Fucales) in south-eastern Australia. Journal of Experimental Marine Biology and Ecology 225:285-300.

Thomsen, M. S., T. Wernberg, A. Altieri, F. Tuva, D. Gulbransen, K. J. McGlathery, M. Holmer, and B. R. Silliman. 2010. Habitat cascades: the conceptual context and global relevance of facilitation cascades via habitat formation and modification. Integrative and Comparative Biology 50: $158-175$.

Underwood, A. J. 1997. Experiments in ecology: their logical design and interpretation using analysis of variance. Cambridge University Press, Cambridge, UK.

van Hulzen, J. B., J. van Soelen, and T. J. Bouma. 2007. Morphological variation and habitat modification are strongly correlated for the autogenic ecosystem engineer Spartina anglica (common cordgrass). Estuaries and Coasts 30:3-11.

Winer, B. J. 1971. Statistical principles in experimental design. Second edition. McGraw-Hill Kogakusha, Tokyo, Japan.

Womersley, H. B. S. 1987. The marine benthic flora of southern Australia. Volume 2. Phaeophyta and Chrysophyta (Vaucheria). Australian Biological Resources Study, Canberra, Australia.

\section{Supplemental Material}

Appendix A

Photographs of the study organisms and experimental plots (Ecological Archives E094-176-A1).

\section{Appendix B}

ANOVAs examining interacting effects of pneumatophore density and height on algal and epifaunal colonization (Ecological Archives E094-176-A2).

\section{Appendix C}

ANOVAs examining interacting effects of pneumatophore density and algal morphology on algal retention by experimental plots, and the colonization of plots by epifaunal mollusks (Ecological Archives E094-176-A3). 